\title{
Pathology 50 years on
}

\author{
Brett Delahunt $^{1,2}$ and Belinda Neill ${ }^{1}$ \\ ${ }^{1}$ Pathology Editorial Office, Royal College of Pathologists of Australasia, Durham Hall, \\ Surry Hills, NSW, Australia; and ${ }^{2}$ Department of Pathology and Molecular Medicine, \\ Wellington School of Medicine and Health Sciences, University of Otago, Wellington, \\ New Zealand
}

This issue of Pathology marks a milestone as the journal enters its 50th year of publication. Pathology commenced in a modest fashion in 1969, being established as an A5 sized quarterly. Interestingly, the focus of the earlier volumes of the journal was on experimental pathology, with six of the eleven manuscripts in the first issue relating to non-human subjects. Over the years the journal has developed a strong clinical focus, publishing articles concerning all of the disciplines of pathology. In parallel with this, Pathology has developed an increasingly international profile, having evolved from a national/regional publication into a truly international journal, as evidenced by the membership of the editorial board and the wide geographic diversity of the authorship. While the journal started off in a modest fashion, it has been able to expand and flourish, adapting to changes in both the academic and professional environment and, to date, 4629 manuscripts, including 3080 original articles/reviews and an additional 1334 abstracts have been published.

A highlight of Pathology has been the evolution of the special theme issue of the journal which became a permanent fixture in 2002. In the early years this focused upon organ systems, with manuscripts restricted to specific topics in anatomical pathology. More recently the focus of the special issue has been expanded to include molecular pathology (2011), biochemical screening for disease (2012), and microbiology (2015). All of the special issues in anatomical pathology have been organ/topic-based and to date, there has not been a general anatomical pathology theme issue. In view of this it was decided that issue 1 of volume 50 would incorporate a wide spectrum of anatomical pathology and would consist of a series of in-depth articles relating to key topics and/or cutting edge areas of histopathology.

This special issue consists of nine reviews covering gynaecological, dermatological, renal, soft tissue, gastrointestinal, prostatic, lymphoproliferative, testicular, breast and endocrine pathology.

In the first article, Dr Robert Young gives a detailed description of the microscopic features and differential diagnoses of sex cord stromal tumours of the ovary. ${ }^{1} \mathrm{He}$ discusses tumours of both epithelial and stromal origin, including adult and juvenile granulosa cell tumour, Sertoli cell tumour, Sertoli-Leydig cell tumour, sex cord tumour with annular tubules, fibroma/thecoma/sclerosing stromal tumour, luteinised thecoma with sclerosing peritonitis and the rare microcystic stromal tumour, and presents useful diagnostic keypoints.

Drs Colebatch and Scolyer, from Royal Prince Alfred Hospital and the Melanoma Institute of Australia, describe the stepwise progression of melanocytic lesions from benign melanocytic naevus to vertical growth phase melanoma and discuss the mutational changes through each of the steps of the pathway. ${ }^{2}$ Importantly, they evaluate the evidence relating to the morphological and genetic features of the intermediate step in the malignant progression of naevus to melanoma.

As a follow-up from the recently published fourth edition of the World Health Organization Classification of Renal Tumours ${ }^{3}$ Drs Eble and Delahunt describe the features of two recently described morphotypes of renal cell neoplasia that are candidates for inclusion in future classifications. ${ }^{4}$ The first of these is designated thyroid-like follicular renal cell carcinoma which, while resembling metastatic follicular carcinoma of the thyroid, has immunohistochemical features more consistent with a renal origin. Oncocytoma-like tumours associated with oncocytosis is the other tumour type discussed in the review. This has some features of tumours associated with the Birt-Hogg-Dubé syndrome, but is lacking folliculin gene mutation.

Drs Schaefer and Fletcher provide an account of recent advances in the field of soft tissue tumours detailing developments in our understanding of the pathology of peripheral nerve sheath, vascular and adipocytic tumours, as well as gastrointestinal stromal tumours, and round cell and myogenic sarcomas. ${ }^{5}$ In this review the authors discuss the genetic and molecular changes associated with several novel tumour entities and further provide descriptions of the diagnostic features including, where appropriate, the morphological spectrum of each tumour type.

The group from University Medical Centre, Utrecht, and The Johns Hopkins University School of Medicine discuss the recent developments in our understanding of the inherited syndromes associated with colorectal cancer. ${ }^{6}$ In particular they describe the diagnostic features and germline mutations associated with Lynch syndrome, familial adenomatous polyposis (classic and attenuated), MUTYH-associated polyposis, Peutz-Jeghers syndrome, juvenile polyposis syndrome, PTEN hamartoma tumour syndrome and serrated polyposis syndrome.

Dr Lars Egevad and colleagues have undertaken a detailed analysis of prognostic factors for prostate cancer and these are presented in the context of the recommendations of the various consensus conferences convened under the auspices of the International Society of Urological Pathology. The review also incorporates the recommendations of the eighth edition of the American Joint Committee on Cancer (AJCC) Tumour, Nodes and Metastases (TNM) staging system 
released in 2017 and provides a contemporary list of prognostic factor of relevance to the practising clinician.

Drs Li, Young and Medeiros provide a comprehensive review of diffuse large B-cell lymphomas. ${ }^{8}$ In this they discuss the classification, clinical features, morphology, immunotyping and genetics, including gene expression profiling and next generation sequencing. In addition, they present a broad outline of the diagnostic work-up and treatment strategies for this tumour group. They also discuss prognostic assessment in the context of the various tumour subgroups that are currently recognised.

The diagnosis of seminoma and other germ cell tumours is becomingly increasingly complicated by our growing understanding of the morphological breadth of atypical forms of these tumours. In the eighth article of this issue of the journal, atypical patterns of seminoma, as well as mimics of seminoma and other testicular malignancies, are discussed by $\mathrm{Dr}$ Ulbright from Indiana University. ${ }^{9}$ In addition, he provides a detailed account of the spectrum of histological features seen in germ cell tumours following chemotherapy. He also gives advice as to how many of the observed changes should be interpreted with respect to determining the necessity for additional treatment and predicting subsequent outcome.

Drs Rakha and Ellis detail the pathology of papillary lesions of the breast with an emphasis on diagnostic features and prognosis. ${ }^{10}$ They present a contemporary classification of papillary lesions and discuss the differential diagnoses as well as histopathological features of diagnostic utility.

In the last of our review articles, Drs Asa and Mete of the University of Toronto provide an oversight into the evolution of our understanding of endocrine pathology. ${ }^{11}$ They highlight historical milestones and describe the three major groups of tissues that are now considered to constitute the endocrine system. They also discuss the impact of molecular biology on the classification of endocrine disease and look towards the future as this specialised area of pathology continues to evolve.
We are most grateful to the contributors who readily replied to our request to submit a manuscript in celebration of this special anniversary and for their enthusiasm to embrace this milestone for the journal. Any milestone is, by its very nature, important but in reality it is just another step on the path and we look forward to the future as the journal continues to support the practice of pathology internationally.

Address for correspondence: Prof Brett Delahunt, Department of Pathology and Molecular Medicine, Wellington School of Medicine and Health Sciences, University of Otago, Wellington, PO Box 7343, Wellington South, New Zealand. E-mail: brett.delahunt@otago.ac.nz

\section{References}

1. Young RH. Ovarian sex cord-stromal tumours and their mimics. Pathology 2018; 50: 5-15.

2. Colbatch AJ, Scolyer RA. Trajectories of premalignancy during the journey from melanocyte to melanoma. Pathology 2018; 50: 16-23.

3. World Health Organization. Classification of Tumours of the Urinary System and Male Genital Organs. 4th ed. Lyon: IARC, 2016.

4. Eble JN, Delahunt B. Emerging entities in renal cell neoplasia: thyroidlike follicular renal cell carcinoma and multifocal oncocytoma-like tumours associated with oncocytosis. Pathology 2018; 50: 24-36.

5. Schaefer I-M, Fletcher CDM. Recent advances in the diagnosis of soft tissue tumours. Pathology 2018; 50: 37-48.

6. Ma H, Brosens LAA, Offerhaus GJA, Giardiello FM, De Leng WWJ, Montgomery EA. Pathology and genetics of hereditary colorectal cancer. Pathology 2018; 50: 49-59.

7. Egevad L, Delahunt B, Kristiansen G, Samaratunga H, Varma M. Contemporary prognostic indicators for prostate cancer incorporating international society of urological pathology recommendations. Pathology 2018; 50: 60-73.

8. Li S, Young KH, Medeiros LJ. Diffuse large B-cell lymphoma. Pathology 2018; 50: 74-87.

9. Ulbright TM. Pitfalls in the interpretation of specimens from patients with testicular tumours, with an emphasis on variant morphologies. Pathology 2018; 50: 88-99.

10. Rakha EA, Ellis IO. Diagnostic challenges in papillary lesions of the breast. Pathology 2018; 50: 100-10.

11. Asa SL, Mete O. Endocrine pathology: past, present and future. Pathology 2018; 50: 111-8. 